\title{
The Effect of Pulse Sequence Parameters and Contrast Agent Dose on Percentage Signal Recovery in DSC-MRI: Implications for Clinical Applications
}

\author{
J.L. Boxerman, E.S. Paulson, M.A. Prah, and K.M. Schmainda
}

\begin{abstract}
BACKGROUND AND PURPOSE: Both technical and pathophysiologic factors affect PSR in DSC-MR imaging. We aimed to determine how TE, flip angle $(\alpha)$, and contrast dose impact PSR in high-grade gliomas.
\end{abstract}

MATERIALS AND METHODS: We retrospectively computed PSR maps for 22 patients with high-grade gliomas, comparing 3 DSC-MR imaging methods by using single-dose gadodiamide without preload administration: $\mathrm{A}(n=7), \alpha=35^{\circ}, \mathrm{TE}=54 \mathrm{~ms} ; \mathrm{B}(n=5), \alpha=72^{\circ}, \mathrm{TE}=$ $30 \mathrm{~ms} ; \mathrm{C}(n=10), \alpha=90^{\circ}, \mathrm{TE}=30 \mathrm{~ms}$. Methods A-C served as preload for subsequent dynamic imaging using method D (method $C$ parameters but with double-dose contrast). We compared first- and second-injection tumor PSR for methods $C$ and D (paired $t$ test) and tumor PSR for both injections grouped by the first-injection acquisition method (3-group nonparametric 1-way ANOVA). We compared PSR in tumor and normal brain for each first- and second-injection method group (paired $t$ test).

RESULTS: First-injection PSR in tumor and normal brain differed significantly for methods $\mathrm{B}(P=.01)$ and $\mathrm{C}(P=.05)$, but not $\mathrm{A}(P=.71)$. First-injection tumor PSR increased with Tl weighting with a significant main effect of method groupings $(P=.0012)$, but there was no significant main effect for first-injection normal brain $(P=.93)$, or second-injection tumor $(P=.95)$ or normal brain $(P=.13)$. In patients scanned with methods $C$ and D, first-injection PSR significantly exceeded second-injection PSR for tumor $(P=.037)$ and normal brain $(P<$ $.001)$.

CONCLUSIONS: PSR strongly depends on the T1 weighting of DSC-MR imaging, including pulse sequence (TE, $\alpha$ ) and contrast agent (dose, preload) parameters, with implications for protocol design and the interpretation and comparison of PSR values across tumor types and imaging centers.

ABBREVIATIONS: DSC = dynamic susceptibility contrast; GBM = glioblastoma multiforme; PSR = percentage signal recovery; rCBV = relative cerebral blood volume

Eirst-pass transient signal loss is the fundamental feature of DSC-MR imaging signal-time curves and the principal determinant of rCBV estimates. However, postbolus signal depression or elevation is frequently observed and represents failure of the signal-time curve, $\mathrm{S}(\mathrm{t})$, and the corresponding relaxivity-time

Received August 16, 2012; accepted after revision September 14.

From the Department of Diagnostic Imaging (J.L.B.), Rhode Island Hospital, Providence, Rhode Island; Alpert Medical School of Brown University (I.L.B.), Providence, Rhode Island; and Departments of Radiation Oncology (E.S.P.), Radiology (E.S.P., M.A.P., K.M.S.), and Biophysics (K.M.S.), Medical College of Wisconsin, Milwaukee, Wisconsin.

This work was supported by NIH/National Cancer Institute RO1 CA082500, Advancing a Healthier Wisconsin, and Medical College of Wisconsin Translational Brain Tumor Research Program.

Please address correspondence to Jerrold L. Boxerman, MD, PhD, Department of Diagnostic Imaging, Rhode Island Hospital, 593 Eddy St, Providence, RI 02903; email: jboxerman@lifespan.org

- Indicates open access to non-subscribers at www.ajnr.org

http://dx.doi.org/10.3174/ajnr.A3477 curve, $\Delta \mathrm{R} 2^{*}(\mathrm{t})$, to return to the prebolus baseline as expected in idealized tracer kinetics. The variable return of the postbolus signal to the prebolus baseline has been termed "percentage signal recovery" and was proposed as a tool for differentiating nonenhancing gliomas and non-neoplastic lesions ${ }^{1}$; progressive metastatic disease from radiation injury after stereotactic radiosurgery $^{2,3}$; recurrent GBM from radiation necrosis ${ }^{4}$; and common intra-axial brain neoplasms such as metastasis, high-grade glioma, and lymphoma. ${ }^{5-8}$

Although PSR has promise for elucidating tumor biology, its etiology is poorly characterized and likely the complex multifactorial interplay of technical (contrast dose, DSC-MR imaging pulse sequence) and pathophysiologic (vascular permeability, tumor cell volume fraction) factors. A spectrum of pulse-sequence parameters with low (long $\mathrm{TE}^{3}$ ), intermediate (low flip angle, intermediate $\mathrm{TE}^{2,4,5}$ and high flip angle, intermediate $\mathrm{TE}^{1,7}$ ), and high (high flip angle, short $\mathrm{TE}^{6}$ ) $\mathrm{T} 1$ weighting have been published for quantifying PSR, with some conflicting inconsistent 
results. Although PSR studies have typically used single-dose contrast without preload, PSR may be evaluated with $\mathrm{rCBV}$, for which higher contrast dose and preload administration for reducing $\mathrm{T} 1$ leakage contamination are beneficial ${ }^{9,10}$; the dependence of PSR on contrast dose and preload is therefore important. For instance, elevated postbolus relaxivity is observed following second contrast agent injections in normal brain ${ }^{11}$ and brain tumors having BBB disruption, especially following preload administration that diminishes T1 leakage effects. ${ }^{12}$ An understanding of the dependence of postbolus signal on these nonphysiologic factors is important for designing protocols that accentuate physiology-dependent differences in PSR and for interpreting PSR results in the clinical setting.

In this study, we aimed to determine how TE, flip angle, and contrast agent dose in DSC-MR imaging impact PSR and to explain why there may be discrepancies in reported PSR values for similar tumors. We hypothesized that PSR depends, in part, on the T1 weighting of the pulse sequence and the total dose of administered contrast agent, including preload. Specifically, at low contrast agent doses, postbolus signal can be dominated by T1 shortening in the intravascular (normal brain) and/or extravascular extracellular space (tumor) when a T1-weighted pulse sequence is used, yielding relatively high PSR. However, with reduced T1 weighting, increased T2* weighting, and increased contrast agent dose, $\mathrm{T} 2^{\star}$ effects can dominate, with substantially smaller PSR. The choice of pulse-sequence parameters (TE, flip angle) and contrast agent dose (administration of preload), which impact the accuracy of rCBV estimation, ${ }^{9,10}$ also has implications for PSR estimates, and understanding this relationship is important for properly selecting PSR imaging parameters, interpreting PSR results, and comparing values across tumor types and imaging centers.

\section{MATERIALS AND METHODS \\ Patient Selection}

We retrospectively analyzed DSC-MR imaging examinations for patients with a cerebral glioma who were previously recruited for perfusion-weighted imaging as a supplement to conventional contrast-enhanced brain MR imaging. Inclusion and exclusion criteria are described in Paulson and Schmainda. ${ }^{12}$ All patients provided informed written consent under Health Insurance Portability and Accountability Act - compliant guidelines established by the institutional review board at the Medical College of Wisconsin. Tumors were classified and graded according to the World Health Organization 1993 Guidelines.

\section{MR Imaging}

All studies were performed at 1.5T (CVi; GE Healthcare, Milwaukee, Wisconsin) with commercial gradients and phased-array radio-frequency coils. To assess the dependence of PSR on pulsesequence parameters, we compared 3 common DSC-MR imaging-acquisition methods with increasing degrees of $\mathrm{T} 1$ weighting, each by using single-dose $(0.1 \mathrm{mmol} / \mathrm{kg})$ gadodiamide (Omniscan; Nycomed Amersham, Princeton, New Jersey) without preload contrast administration: method A $(n=7)$, single echo with a low flip angle $\left(\alpha=35^{\circ}\right)$ and long TE (54 ms) yielding minimum T1 sensitivity ${ }^{13-15}$; method $\mathrm{B}(n=5)$, second echo of a dual-echo spiral acquisition (first-echo $\mathrm{TE}=3.3 \mathrm{~ms}$ ) with an intermediate flip angle $\left(\alpha=72^{\circ}\right)$ and short TE (30 ms), yielding
Table 1: Summary of DSC-MRI pulse-sequence parameters compared in this study

\begin{tabular}{lccccc}
\hline $\begin{array}{c}\text { MRI } \\
\text { Method }\end{array}$ & No. & $\begin{array}{c}\text { Preload } \\
(\mathbf{m m o l} / \mathbf{k g})\end{array}$ & $\begin{array}{c}\text { Bolus } \\
(\mathbf{m m o l} / \mathbf{k g})\end{array}$ & $\boldsymbol{\alpha}\left(^{\circ}\right)$ & TE (ms) \\
\hline A & 7 & 0 & 0.1 & 35 & 54 \\
$B^{\mathrm{a}}$ & 5 & 0 & 0.1 & 72 & 30 \\
C & 10 & 0 & 0.1 & 90 & 30 \\
D & 22 & 0.1 & 0.2 & 90 & 30 \\
\hline
\end{tabular}

a Second echo of a dual-echo acquisition (first-echo TE = $3.3 \mathrm{~ms}$ ); other methods were single-echo acquisitions. All methods used gradient-echo EPI.

intermediate T1 sensitivity ${ }^{16,17}$; and method C $(n=10)$, single echo with high flip angle $\left(\alpha=90^{\circ}\right)$ and short TE (30 ms), yielding the greatest T1 sensitivity. ${ }^{9,18,19}$ Method D, applied to all patients, was identical to method $\mathrm{C}$ but used double-dose contrast $(0.2$ $\mathrm{mmol} / \mathrm{kg}$ ) following the $0.1 \mathrm{mmol} / \mathrm{kg}$ preload that served as the bolus for dynamic imaging in methods A-C. Table 1 summarizes the pulse sequence parameters for each method. For single-echo acquisitions, a lipid-suppressed single-shot gradient-echo EPI pulse sequence acquired images for 1 minute before and 2 minutes after bolus injection of contrast agent at $3-5 \mathrm{~mL} / \mathrm{s}\left(\mathrm{FOV}=24 \mathrm{~cm}^{2}\right.$, matrix $=64 \times 64, \mathrm{TR}=1000 \mathrm{~ms}$, section thickness $=5 \mathrm{~mm}$, intersection gap $=1.5 \mathrm{~mm}, 12$ sections). For the dual-echo acquisition, a custom lipid-suppressed single-shot dual-echo gradient-echo spiralout sequence was used with the same general parameters as the single-echo acquisitions. ${ }^{20}$ Following DSC-MR imaging, postcontrast T1-weighted spin-echo images were acquired $(\mathrm{TR}=450 \mathrm{~ms}, \mathrm{TE}=$ $11 \mathrm{~ms}, \mathrm{NEX}=2$, matrix $=256 \times 256$ ).

\section{PSR Computation}

For each patient, we constructed voxelwise PSR maps from the time course data for both contrast agent injections by using a standard technique ${ }^{7}$ :

$$
\operatorname{PSR}=\left(S_{1}-S_{\text {min }}\right) /\left(S_{o}-S_{\text {min }}\right) \times 100 \%,
$$

where baseline $\left(S_{o}\right)$ and postbolus signal intensity $\left(S_{1}\right)$ equals the average signal intensity of time points $25-55$ and $125-155$, respectively, and $S_{\text {min }}$ is the minimum signal intensity within the firstpass bolus. We selected a region of interest $\left(30-50 \mathrm{~mm}^{2}\right)$ for enhancing tumor, excluding areas of necrosis and nontumor macrovessels; and a region of interest of identical size was specified for contralateral normal-appearing brain. The same ROIs for each patient were used for both contrast agent injections. Mean and standard error of the PSR within tumor and normal brain ROIs were computed for both injections for each patient by translating each region of interest onto the appropriate PSR map.

\section{Statistical Methods}

Statistical analysis was performed by using GraphPad Prism, Version 5.0d (GraphPad Software, LA Jolla, California). To investigate the dependence of PSR on contrast agent dose and preload, we compared mean tumor PSR from the first and second injections (differing in bolus dose and the presence of preload) in patients scanned with the otherwise identical methods $C$ (first injection) and $\mathrm{D}$ (second injection) by using a 2-tailed paired $t$ test. We repeated the analysis for mean PSR in normal brain. To investigate the dependence of PSR on pulse-sequence parameters, we compared first-injection mean tumor PSR in patients grouped by acquisition methods $\mathrm{A}-\mathrm{C}$ by using a 3-group nonparametric 
Table 2: Mean PSRs grouped by DSC-MRI acquisition method

\begin{tabular}{|c|c|c|c|c|c|c|c|c|c|}
\hline \multirow{3}{*}{$\begin{array}{l}\text { MRI Method }{ }^{a} \\
\text { (Inj1, Inj2) }\end{array}$} & \multirow{2}{*}{\multicolumn{5}{|c|}{ Tumor Characteristics }} & \multicolumn{4}{|c|}{ Mean PSR $(\% \pm$ SEM) } \\
\hline & & & & & & \multicolumn{2}{|c|}{ 1st Injection } & \multicolumn{2}{|c|}{ 2nd Injection } \\
\hline & No. & GIII & GIV & New & $\operatorname{Rec}$ & Tumor & Normal & Tumor & Normal \\
\hline$A, D$ & 7 & 1 & 6 & 1 & 6 & $91 \pm 10$ & $96 \pm 5$ & $81 \pm 10$ & $96 \pm 8$ \\
\hline$B, D$ & 5 & 1 & 4 & 0 & 5 & $217 \pm 32$ & $103 \pm 14$ & $76 \pm 8$ & $78 \pm 4$ \\
\hline C, D & 10 & 5 & 5 & 7 & 3 & $372 \pm 122$ & $95 \pm 2$ & $79 \pm 8$ & $80 \pm 2$ \\
\hline
\end{tabular}

Note:-SEM indicates standard error of the mean; Inj1, injection 1; Inj2, injection 2; GIII, grade III gliomas; GIV, grade IV gliomas; New, treatment-naïve or residual postoperative; Rec, recurrent (after conventional chemoradiation). ${ }^{\text {a } M R I ~ m e t h o d s ~ r e f e r ~ t o ~ f i r s t-i n j e c t i o n ~ a n d ~ s e c o n d-i n j e c t i o n ~ a c q u i s i t i o n ~ t e c h n i q u e s ~ f o r ~ e a c h ~ p a t i e n t ~(T a b l e ~ 1) . ~}$

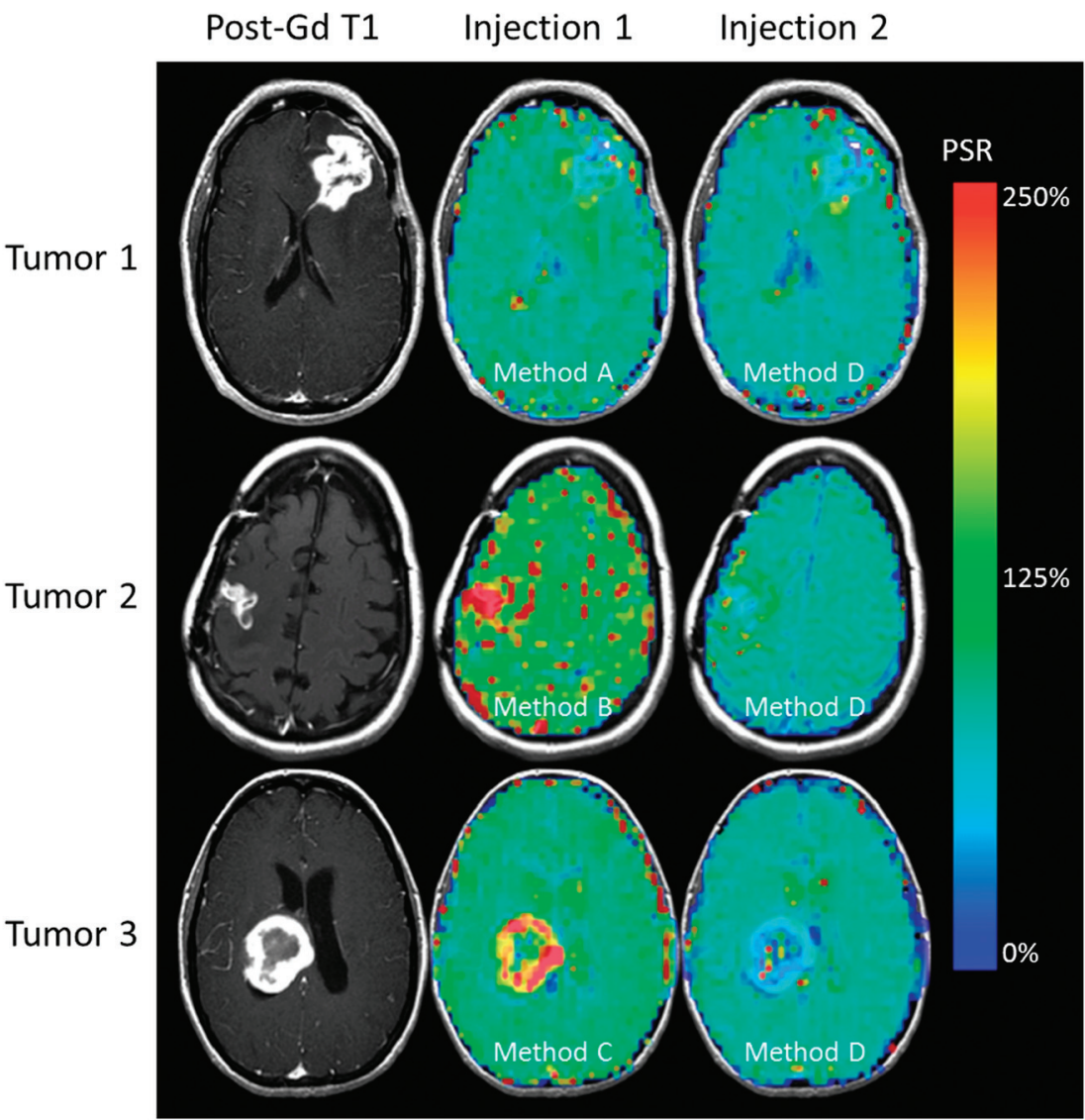

FIG 1. Three sample tumors (first column) with corresponding first-injection (middle column) and second-injection (third column) PSR maps. First-injection PSR progressively increases with increasing $\mathrm{Tl}$ weighting of methods A (first row), B (second row), and C (third row). Secondinjection maps (all by using method D) have lower PSR with poor contrast between tumor and normal brain, despite flip angles and TEs identical to those in method C. Preload administration decreases $\mathrm{Tl}$ sensitivity and PSR contrast, regardless of flip angle and TE.

1-way ANOVA (Kruskal-Wallis test), and we performed follow-up group comparisons by using the Dunn multiple comparison test. We repeated this analysis for second-injection mean tumor PSR, and for first- and second-injection mean PSR in normal brain. We also compared mean PSR in tumor and normal brain for each first- and second-injection method group, by using a 2-tailed paired $t$ test. For all tests, we assumed a statistically significant threshold of $P=.05$.

\section{RESULTS}

\section{Study Cohort}

Twenty-two patients (12 men, 10 women; $21-76$ years of age; mean age, 49 years) with high-grade gliomas were enrolled. Grade III tu- mors included new $(n=1)$ and recurrent $(n=2)$ anaplastic astrocytomas, recurrent anaplastic mixed gliomas $(n=2)$, new $(n=$ $1)$ and recurrent $(n=1)$ anaplastic oligodendrogliomas, and malignant glioneuronal tumor $(n=1)$. Grade IV tumors included newly diagnosed $(n=5)$, recurrent $(n=7)$, and residual GBMs $(n=2)$. At the time of DSC-MR imaging, 15 patients were being treated with steroids. Table 2 summarizes the tumor characteristics grouped by the first-injection acquisition method, as well as intragroup mean and standard error in tumor and normal brain for both first (methods A-C) and second (all method D) injections. For the first injection (no preload), methods A-C have increasing $\mathrm{T} 1$ weighting (increasing flip angle, decreasing TE), with increasing PSR in tumors that substantially exceeds $100 \%$ for methods $\mathrm{B}-\mathrm{C}$ and equivalent $\mathrm{PSR} \approx 100 \%$ for all methods in normal brain. For the second injection (with preload), all 3 groups used method D, with essentially equivalent PSRs in tumor $(76 \%-81 \%)$ and normal brain (78\%-96\%).

Figure 1 illustrates 3 cases, each with contrast-enhanced tumor (first column) and corresponding first-injection (middle column) and second-injection (third column) PSR maps. First-injection methods A (first row), B (second row), and C (third row) represent increasing $\mathrm{T} 1$ weighting, with progressively higher PSR values. With increasing flip angles and decreasing TEs, T1 sensitivity and PSR increase. The secondinjection maps (all acquired with method D) demonstrate poor PSR contrast with substantially lower PSR. Although method D has flip angles and TEs identical to those in method $\mathrm{C}$, which yields high first-injection PSR, preload contrast administration substantially diminishes $\mathrm{T} 1$ effects, reducing tumor PSR to levels seen with the first-injection method A. In the presence of preload contrast administration, T1 sensitivity and PSR decrease, regardless of flip angle and TE.

\section{Dependence of PSR on Contrast Agent Dose and Timing}

Figure $2 A$ illustrates sample first-injection signal-time curves for tumor and normal brain acquired with method C (heavily $\mathrm{T} 1$ weighted; $\alpha=90^{\circ}$, and TE $=30 \mathrm{~ms}$ ). Without preload, there is high postbolus signal intensity and PSR $>100 \%$ for tumor, with PSR $\approx 100 \%$ for normal brain. Figure $2 B$ illustrates the corresponding second-injection signal-time curves for identical ROIs and acquisition parameters (method D). Preload diminishes the T1 effects in tumor from extravasated contrast, yielding reduced postbolus signal and PSR $<100 \%$. In normal brain, post- 


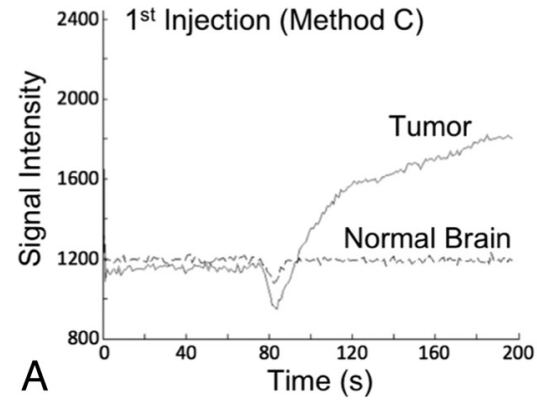

Tumor

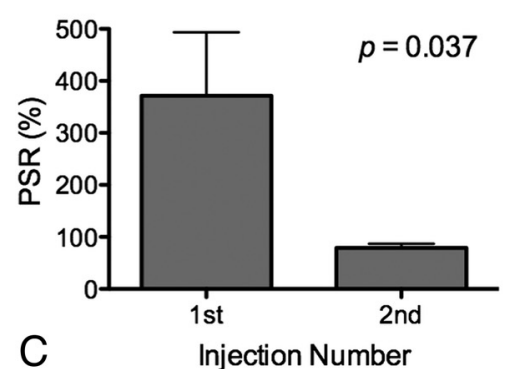

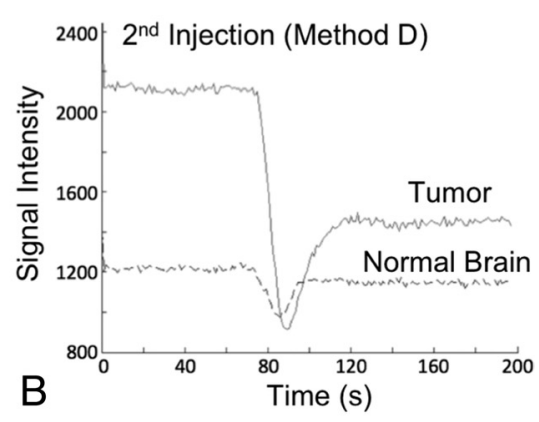

Normal Brain

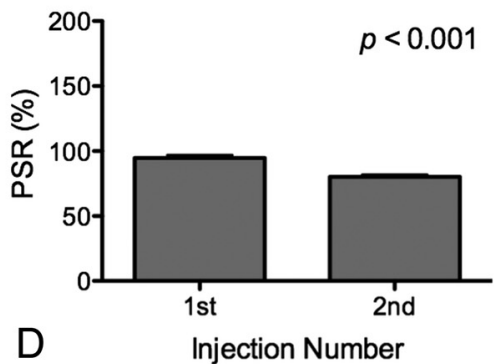

FIG 2. Sample first-injection $(A)$ and second-injection $(B)$ signal-time curves for tumor and normal brain by using methods $\mathrm{C}$ and $\mathrm{D}\left(\alpha=90^{\circ}, \mathrm{TE}=30 \mathrm{~ms}\right)$. Without preload $(A)$, tumor PSR is $>100 \%$, whereas with preload $(B)$, tumor PSR is $<100 \%$. First-injection PSR (method C) is significantly larger than second-injection PSR (method D) for both tumor $(C ; P=.037)$ and normal brain $(D ; P<.001)$ by using the 2 -tailed paired $t$ test.

bolus signal does not quite reach baseline, with PSR $<100 \%$. Figure $2 C,-D$ compares mean PSR on the first (method $C$ ) and second (method D) injections, both with $\alpha=90^{\circ}$ and TE $=30 \mathrm{~ms}$, in tumor and normal brain, respectively. First-injection PSR significantly exceeds second-injection PSR for both tumor $(P=$ $.037)$ and normal brain $(P<.001)$ by using the 2 -tailed paired $t$ test. These results demonstrate that PSR can differ significantly, depending on whether preload contrast is administered.

\section{Dependence of PSR on MR Imaging Acquisition \\ Parameters}

For the first injection, there was no significant difference between mean PSR in tumor and normal brain for method $\mathrm{A}(P=.71)$, but there was a significant difference for methods $\mathrm{B}(P=.01)$ and $\mathrm{C}$ $(P=.05)$. For the second-injection method (method D), there was no significant difference in patients grouped by the first-injection method A $(P=.15), \mathrm{B}(P=.71)$, or $\mathrm{C}(P=.85)$.

Figure 3 compares mean PSR values in tumor and normal brain for first-injection methods A-C (Fig 3A, $-C$ ) and for second-injection method $\mathrm{D}$ (Fig $3 B,-D$ ) with patients grouped by the first-injection method. For the first injection in tumors, there was a significant main effect of method groupings on PSR by the Kruskal-Wallis test $(P=.0012$; Fig $3 A$ ), with mean PSR monotonically increasing with T1 weighting. Follow-up group comparisons (Dunn multiple comparison test) revealed that method A tumors had significantly lower PSR than method B and method C tumors; there was no significant difference in PSR between method B tumors and method $\mathrm{C}$ tumors. For normal brain, there was no significant main effect of first-injection acquisitionmethod groupings on PSR by the Kruskal-Wallis test $(P=.93$;

Fig 3C). For the second injection, all patients were scanned with method D, regardless of first-injection methodology. There was no significant main effect of the first-injection acquisition-method groupings on second-injection PSR by the Kruskal-Wallis test for tumor $(P=.95$; Fig $3 B)$ or normal brain $(P=.13$; Fig $3 D)$.

\section{DISCUSSION}

PSR compares postbolus DSC-MR imaging signal recovery with prebolus baseline and has the potential for differentiating neoplastic and non-neoplastic primary lesions ${ }^{1}$; progressive primary ${ }^{4}$ or metastatic $^{2,3}$ neoplasms from radiation injury following radiosurgery; and common intra-axial brain tumors including metastasis, high-grade glioma, and lymphoma. ${ }^{5-8}$ However, a formal study of the technical factors contributing to PSR has not been performed, to our knowledge. This analysis is important for understanding the degree to which PSR variations from technical factors can overshadow desired variations due to underlying pathophysiology.

The postbolus DSC-MR imaging signal has T1 and T2* contributions resulting from the complex interplay of biophysical factors, including vascular, cell, and interstitial volume fractions and vascular permeability, which govern the relative contrast agent concentrations in the vascular and extravascular extracellular space. ${ }^{21} \mathrm{~T} 2^{*}$ relaxivity arises from the superposition of the "positive" susceptibility effect due to intravascular compartmentalization of contrast agent within tumor microvasculature and the "inverse" susceptibility effect, due to exclusion from cells of extravasated contrast agent within the extravascular extracellular space, and depends on the proton diffusion rate within tissue. T1 relaxivity arises from dipole-dipole interactions between protons and contrast agent weighted by relative concentrations and volumes of distribution in the intravascular and extravascular extracellular compartments. Presumably, differing tumor-specific cell densities, vascular permeabilities, and vascular volume fractions in GBM, lymphoma, and metastases yield unique PSR signatures permitting their differentiation, ${ }^{7}$ with analogous physiologic differences accounting for discrepant PSR in tumor, radiation necrosis, and other non-neoplastic processes.

Pulse-sequence parameters also affect PSR by conferring different T1 and T2* weighting on DSC-MR imaging signal, and our results indicate that this effect can be profound. For enhancing tumors with extravasated contrast, increased T1 weighting with relatively low $\mathrm{T}^{\star}$ weighting yields higher PSR estimates, which can substantially exceed $100 \%$ for high-flip-angle low-TE acquisitions and TR $=1000 \mathrm{~ms}$ (Fig 3A). In fact, our signal-time curves for some high-grade gliomas by using method $\mathrm{C}$ with high $\mathrm{T} 1$ weighting (Fig 2A) look nearly identical to those for lymphoma by Mangla et al (Fig 1B), ${ }^{7}$ exemplifying how PSR dependence on 


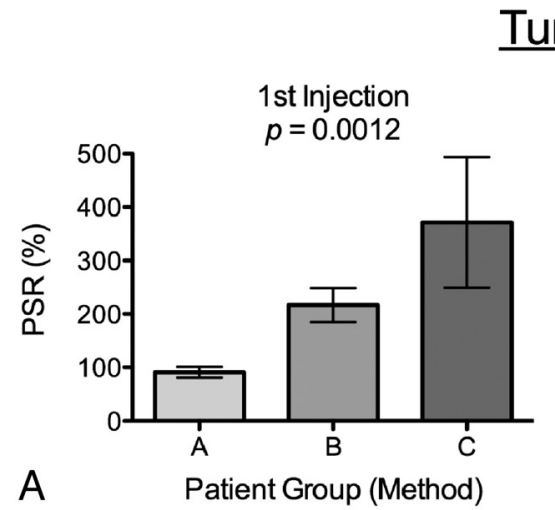

$\underline{\text { Tumor }}$

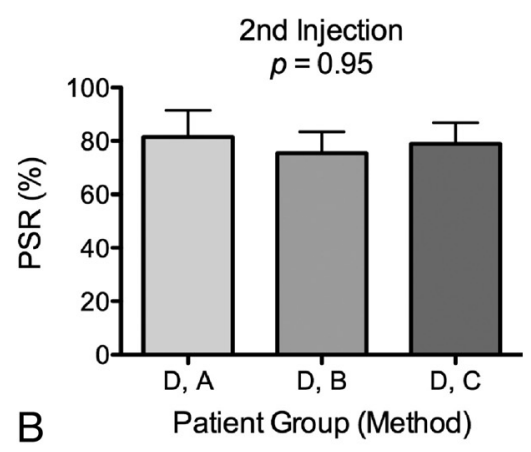

$\underline{\text { Normal Brain }}$
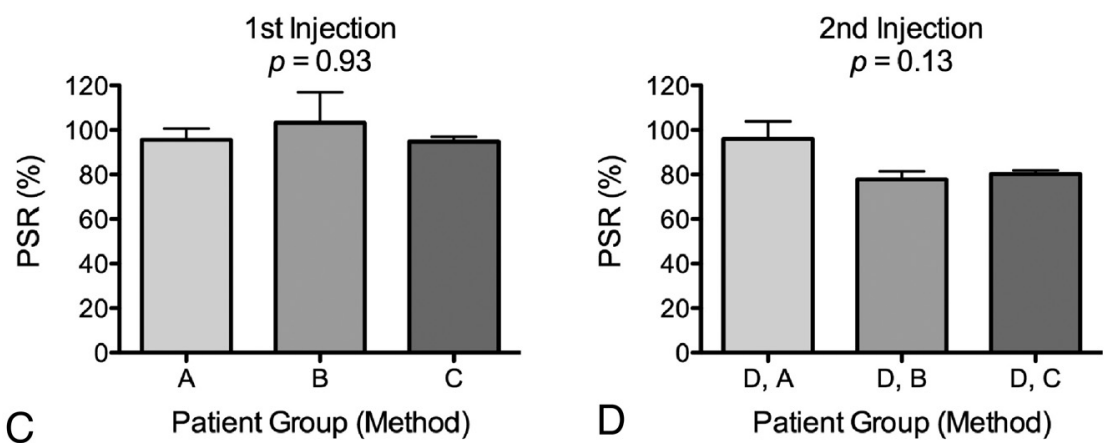

FIG 3. Comparison of PSR in tumor $(A)$ and normal brain $(C)$ for methods $A-C$ and in tumor $(B)$ and normal brain $(D)$ for second-injection method $D$ grouped by first-injection method. There is a significant main effect of method groupings on first-injection tumor PSR ( $A ; P=.0012)$, with PSR monotonically increasing with TI weighting and no significant main effect on first-injection normal brain PSR ( $C ; P=.93)$. There is no significant main effect of first-injection method groupings on second-injection PSR for tumor $(B ; P=.95)$ or normal brain $(D ; P=.13)$. injection. An alternative approach uses a dual-echo acquisition during single contrast agent injection, when parameters for the second echo are selected to provide $\mathrm{T} 1$ and $\mathrm{T} 2{ }^{\star}$ weighting for PSR derivation and the first echo is used with the second echo to eliminate $\mathrm{T} 1$ weighting for $\mathrm{rCBV}$ mapping. ${ }^{20}$

Our measured PSR values match the literature values for comparable acquisition parameters. We found the mean first-injection tumor PSR with method A to be $91 \%$, in good agreement with mean values of $81 \%$ observed by Cha et $\mathrm{al}^{5}$ and $80 \%$ by Barajas et $\mathrm{al}^{4}$, both obtained with identical flip angles and TEs for GBMs. Our slightly higher value may result from a mix of high-grade gliomas in our population, shorter TRs (1000 ms versus 1000 $1250 \mathrm{~ms}$ ), and more baseline points, which may lower the baseline estimate due to reduced non-steady-state contamination.

Our study does not investigate the impact of TR on PSR. We used TR $=1000 \mathrm{~ms}$ for all methods; this was our protocol for DSC examinations geared toward rCBV estimation, because the high temporal resolution of $\Delta \mathrm{R} 2^{*}(\mathrm{t})$ improves $\mathrm{rCBV}$ map $\mathrm{SNR}^{22}$ Longer TR confers greater $\mathrm{T} 2$ weighting, and this effect can be substantial. For example, TE and $\alpha$ for our method C are identical to those used by Liao et al, ${ }^{6}$ but their mean PSR in GBM was $93 \%$ for TR = pulse sequence can confound clinical interpretations of PSR. Preload administration eliminates PSR dependence on T1 weighting even in tumors (Fig 3B) by saturating the baseline extravascular extracellular compartment T1-weighted signal intensity, thereby diminishing T1-induced increased signal during second-injection DSC-MR imaging. It may also reduce the gradient of contrast efflux. This effect is desirable for rCBV estimation, in which preload and postprocessing algorithms reduce error in rCBV estimates by minimizing T1 contamination, ${ }^{9,10}$ but it is suboptimal for PSR mapping because T1 sensitivity plays a pivotal role in establishing unique physiology-dependent PSR signatures of different neoplastic and non-neoplastic enhancing lesions. For instance, there was no significant difference in PSR between tumor and normal brain for first-injection method A with low T1 weighting, and second-injection postpreload PSR for tumor and normal brain was not significantly different, even for the high T1 weighting of method D.

Optimal DSC-MR imaging pulse sequences for PSR discrimination that have substantial T1 weighting may, therefore, be suboptimal for accurate CBV imaging, in which contaminating T1 effects should be minimized with preload and a low flip angle. There are several approaches to a comprehensive DSC protocol. PSR imaging could be performed dynamically on the preload injection with subsequent dynamic CBV imaging on the second
$1440 \mathrm{~ms}$, compared with our value of $372 \%$. In fact, the morphology of our $\mathrm{S}(\mathrm{t})$ curve and corresponding PSR value for these parameters are closer to those found by Liao et al for lymphoma than for GBM. Furthermore, our measured first-injection PSR for method A of 91\% is similar to the mean GBM PSR of 78\% found by Mangla et $\mathrm{al}^{7}$ and the mean nonenhancing glioma PSR of $88 \%$ found by Liu et al ${ }^{1}$ for $\mathrm{TE}=50 \mathrm{~ms}$, with the substantially increased $\mathrm{T} 2$ weighting for their $\mathrm{TR}=1500 \mathrm{~ms}$ apparently offsetting the much higher T1 weighting for their $\alpha=80^{\circ}$.

Because pulse-sequence parameters can significantly affect the magnitude of measured PSR, care should be taken when interpreting and comparing PSR results from the literature. For instance, Huang et $\mathrm{al}^{3}$ found no difference in PSR for progressive metastases versus radiation injury by using a heavily T2-weighted acquisition (TR/TE $=2480 / 98 \mathrm{~ms}$ ), whereas Barajas et $\mathrm{al}^{2}$ found a significant difference by using intermediate T1 weighting (TR/ $\mathrm{TE} / \alpha=1250 \mathrm{~ms} / 54 \mathrm{~ms} / 35^{\circ}$ ). Furthermore, mean glioma PSRs between $78 \%$ and $93 \%$ have been reported by using techniques ranging from high $\alpha$, low $\mathrm{TE}^{6}$ to low $\alpha$, intermediate $\mathrm{TE},{ }^{4}$ all with intermediate TRs between 1250 and $1500 \mathrm{~ms}$. Although this range of PSR is smaller than ours obtained with a much smaller TR, there is overlap of the highly T1-weighted comparative GBM data $^{6}$ with our first-injection normal brain PSRs and of lower T1-weighted comparative data with our second-injection normal 
brain PSRs, further emphasizing the sensitivity of PSR estimates to DSC-MR imaging methodology.

This study has several limitations. Our sample population was heterogeneous and included grade III and IV tumors that are both treatment-näive and recurrent, with variable steroid administration; this may introduce some variability. Vascular volume fraction for GBM exceeds that for grade III gliomas, ${ }^{9}$ with cell density and vascular permeability differences; chemoradiation and steroids can affect tumor physiology as well. However, tumor populations were mixed in our 3 method groups, and the aggregate populations are probably similar. Intergroup comparison (Fig 3) should, therefore, emphasize true pulse sequence differences rather than variable underlying tumor physiology. Furthermore, we demonstrated significant differences in PSR due to contrast agent preload (Fig 2) by using a paired nonparametric $t$ test in which each tumor was its own control, and a heterogeneous tumor population would not affect this analysis. Our retrospective data did not permit us to isolate the effects of contrast agent dose versus preload on PSR. To do so would require a comparison, for instance, of single- and double-dose contrast agent for the first injection or single-dose administrations of 2 different contrast agents with different relaxivities.

\section{CONCLUSIONS}

PSR strongly depends on the T1 weighting of the DSC-MR imaging acquisition as determined by pulse sequence (TE, flip angle) and contrast agent (dose and preload) factors. Understanding this relationship is important for properly selecting PSR imaging parameters, interpreting PSR results, and comparing values across tumor types and imaging centers. Because accurate CBV imaging requires minimization of $\mathrm{T} 1$ effects, comprehensive DSC-MR imaging protocols that aim to measure rCBV and PSR for assessing tumor physiology must address these competing factors.

Disclosures: Melissa A. Prah—RELATED: Grant: Medical College of Wisconsin. ${ }^{*}$ Kathleen M. Schmainda—RELATED: Grant: National Institutes of Health (NIH), ${ }^{*}$ UNRELATED: Grants/Grants Pending: NIH, Royalties: Imaging Biometrics LLC, Comments: paid directly to the institution, which, in turn, pays a percentage to the inventors, ${ }^{*}$ Stock/Stock Options: ownership interest in Prism Clinical Imaging Inc, Other: ownership interest in Imaging Biometrics LLC and spouse's salary. * Money paid to the institution.

\section{REFERENCES}

1. Liu X, Kolar B, Tian W, et al. MR perfusion-weighted imaging may help in differentiating between nonenhancing gliomas and nonneoplastic lesions in the cervicomedullary junction. J Magn Reson Imaging 2011;34:196-202

2. Barajas RF, Chang JS, Sneed PK, et al. Distinguishing recurrent intra-axial metastatic tumor from radiation necrosis following gamma knife radiosurgery using dynamic susceptibility-weighted contrast-enhanced perfusion MR imaging. AJNR Am J Neuroradiol 2009;30:367-72

3. Huang J, Wang AM, Shetty A, et al. Differentiation between intraaxial metastatic tumor progression and radiation injury following fractionated radiation therapy or stereotactic radiosurgery using MR spectroscopy, perfusion MR imaging or volume progression modeling. Magn Reson Imaging 2011;29:993-1001

4. Barajas RF Jr, Chang JS, Segal MR, et al. Differentiation of recurrent glioblastoma multiforme from radiation necrosis after external beam radiation therapy with dynamic susceptibilityweighted contrast-enhanced perfusion MR imaging. Radiology 2009;253:486-96

5. Cha S, Lupo JM, Chen MH, et al. Differentiation of glioblastoma multiforme and single brain metastasis by peak height and percentage of signal intensity recovery derived from dynamic susceptibility-weighted contrast-enhanced perfusion MR imaging. AJNR Am J Neuroradiol 2007;28:1078-84

6. Liao W, Liu Y, Wang X, et al. Differentiation of primary central nervous system lymphoma and high-grade glioma with dynamic susceptibility contrast-enhanced perfusion magnetic resonance imaging. Acta Radiol 2009;50:217-25

7. Mangla R, Kolar B, Zhu T, et al. Percentage signal recovery derived from MR dynamic susceptibility contrast imaging is useful to differentiate common enhancing malignant lesions of the brain. AJNR Am J Neuroradiol 2011;32:1004-10

8. Mouthuy N, Cosnard G, Abarca-Quinones J, et al. Multiparametric magnetic resonance imaging to differentiate high-grade gliomas and brain metastases. J Neuroradiol 2012;39:301-07

9. Boxerman JL, Schmainda KM, Weisskoff RM. Relative cerebral blood volume maps corrected for contrast agent extravasation significantly correlate with glioma tumor grade, whereas uncorrected maps do not. AJNR Am J Neuroradiol 2006;27:859-67

10. Boxerman JL, Prah DE, Paulson ES, et al. The role of preload and leakage correction in gadolinium-based cerebral blood volume estimation determined by comparison with MION as a criterion standard. AJNR Am J Neuroradiol 2012;33:1081-87

11. Levin JM, Kaufman MJ, Ross MH, et al. Sequential dynamic susceptibility contrast MR experiments in human brain: residual contrast agent effect, steady state, and hemodynamic perturbation. Magn Reson Med 1995;34:655-63

12. Paulson ES, Schmainda KM. Comparison of dynamic susceptibilityweighted contrast-enhanced MR methods: recommendations for measuring relative cerebral blood volume in brain tumors. Radiology 2008;249:601-13

13. Cha S, Lu S, Johnson G, et al. Dynamic susceptibility contrast MR imaging: correlation of signal intensity changes with cerebral blood volume measurements. J Magn Reson Imaging 2000;11:114-19

14. Law M, Yang S, Babb JS, et al. Comparison of cerebral blood volume and vascular permeability from dynamic susceptibility contrastenhanced perfusion MR imaging with glioma grade. AJNR Am J Neuroradiol 2004;25:746-55

15. Lee MC, Cha S, Chang SM, et al. Dynamic susceptibility contrast perfusion imaging of radiation effects in normal-appearing brain tissue: changes in the first-pass and recirculation phases. J Magn Reson Imaging 2005;21:683-93

16. Vonken EP, van Osch MJ, Bakker CJ, et al. Simultaneous quantitative cerebral perfusion and Gd-DTPA extravasation measurement with dual-echo dynamic susceptibility contrast MRI. Magn Reson Med 2000;43:820-27

17. Uematsu H, Maeda M, Sadato N, et al. Blood volume of gliomas determined by double-echo dynamic perfusion-weighted MR imaging: a preliminary study. AJNR Am J Neuroradiol 2001;22:1915-19

18. Donahue KM, Krouwer HG, Rand SD, et al. Utility of simultaneously acquired gradient-echo and spin-echo cerebral blood volume and morphology maps in brain tumor patients. Magn Reson Med 2000;43: $845-53$

19. Schmainda KM, Rand SD, Joseph AM, et al. Characterization of a first-pass gradient-echo spin-echo method to predict brain tumor grade and angiogenesis. AJNR Am J Neuroradiol 2004;25:1524-32

20. Paulson ES, Prah DE, Schmainda KM. Correction of confounding leakage and residual susceptibility effects in dynamic susceptibility contrast MR imaging using dual-echo SPIRAL. In: Proceedings of the 45th Annual Meeting of the American Society of Neuroradiology and Neuroradiology Education and Research Foundation Symposium, Chicago, Illinois; June 7-14, 2007

21. Quarles CC, Gochberg DF, Gore JC, et al. A theoretical framework to model DSC-MRI data acquired in the presence of contrast agent extravasation. Phys Med Biol 2009;54:5749-66

22. Boxerman JL, Rosen BR, Weisskoff RM. Signal-to-noise analysis of cerebral blood volume maps from dynamic NMR imaging studies. J Magn Reson Imaging 1997; 7:528-37 